\title{
Achromatic diffractive lens written onto a liquid crystal display
}

\author{
A. Márquez \\ Departamento de Física, Ingeniería de Sistemas y Teoría de la Señal, Universidad de Alicante, \\ Apartado 99, 03080 Alicante, Spain \\ C. Iemmi \\ Departamento de Física, Facultad de Ciencias Exactas y Naturales, Universidad de Buenos Aires, \\ 1428 Buenos Aires, Argentina \\ J. Campos and M. J. Yzuel \\ Departamento de Física, Universidad Autónoma de Barcelona, 08193 Bellaterra, Spain
}

\begin{abstract}
Received July 20, 2005; revised September 14, 2005; accepted October 19, 2005
We propose a programmable diffractive lens written onto a liquid crystal display (LCD) that is able to provide equal focal lengths for several wavelengths simultaneously. To achieve this goal it is necessary that the LCD operate in the phase-only regime simultaneously for the different wavelengths. We design the appropriate lens for each wavelength, and then the lenses are spatially multiplexed onto the LCD. Various multiplexing schemes have been analyzed, and the random scheme shows the best performance. We further show the possibility of finely tuning the chromaticity of the focal spot by changing the relative weights of the multiplexing among the various wavelengths. (C) 2006 Optical Society of America

OCIS codes: $050.1970,230.3720,230.6120,220.3620$.
\end{abstract}

Twisted-nematic liquid crystal displays (LCDs) have been proved to be appropriate devices for generating programmable diffractive optical elements (DOEs), and in particular diffractive lenses. ${ }^{1}$ In general the performance of LCDs and programmable DOEs is very sensitive to the wavelength of the incident illumination. In diffractive lenses a change in wavelength produces a change in focal length and may further produce a change in the diffraction efficiency. As a consequence diffractive singlets are designed and applied for monochromatic illumination. Achromatization can be obtained if two or more diffractive singlets are combined, and if refractive achromats are also introduced. ${ }^{2}$ DOEs' capability to perform various functions simultaneously may also be used to achromatize the performance of diffractive singlets. Different multiplexing schemes exist, such as multifunction coding methods ${ }^{3}$ or spatial multiplexing methods. The latter were recently used to extend the depth of field of an imaging system using diffractive lenses. ${ }^{4}$ In this work we show that, by means of a spatial multiplexing scheme, we can design an apochromat diffractive lens, i.e., a lens with the same focal length at three discrete wavelengths $(633,514$, and $458 \mathrm{~nm}$ ). We also show the chromatic properties offered by the simultaneous tunability of the efficiency of the apochromat at these three wavelengths. The diffractive lens is programmed onto a twistednematic LCD whose performance has been optimized for polychromatic illumination. We can think of the above-mentioned three wavelengths as discrete RGB channels whose control allows one to change the chromaticity in an optical system.

A lens with a focal length $f^{\prime}$ can be described as an optical element that introduces, in the paraxial approximation, a quadratic phase onto an incident wavefront. If a diffractive lens designed for a wavelength $\lambda$ is illuminated with a different wavelength $\lambda_{1}$, the focal length shifts to $f_{1}^{\prime}=\left(f^{\prime} \lambda\right) / \lambda_{1}$. Furthermore, if we analyze the diffraction efficiency $\eta$ of the lens for the primary focus we obtain $\eta=\operatorname{sinc}^{2}(1$ $-\Delta \varphi / 2 \pi)$, where $\operatorname{sinc}(x)=\sin (\pi x) / \pi x$ and $\Delta \varphi$ is the maximum phase depth in each ring. In general, the maximum phase depth $\Delta \varphi$ varies with the incident wavelength, thus changing the diffraction efficiency. To overcome the wavelength dependencies of the focal length and the diffraction efficiency we propose in this work to use spatial multiplexing strategies to generate the diffractive lens. Furthermore, we want the lens to be displayed on a LCD to take advantage of the flexibility offered by real-time control from a computer. This requires a phase-only modulation regime with an LCD that is valid for the whole range of wavelengths of interest.

To obtain a phase-only regime for the LCD valid for a wide range of wavelengths we extend the optimization procedure proposed in Ref. 5: now the figure of merit to be optimized takes into account several wavelengths simultaneously that cover the whole visible spectrum. We use a Sony LCD, Model LCX012BL, extracted from a Sony video projector, Model VPL-V500. The LCD is inserted between two sets composed of a polarizer and a wave plate. In Figs. 1(a) and 1(b) we plot the amplitude and the phase shift modulation versus the gray level, respectively, for the three wavelengths considered for the multiplexed lenses in this work $(633,514$, and 458 $\mathrm{nm}$ ). We show both the experimental values (symbols) and the predicted values (solid curves) by our model. ${ }^{5}$ There is very good agreement between these values. One can see that the transmission is very uniform and almost equal for the three wavelengths. 

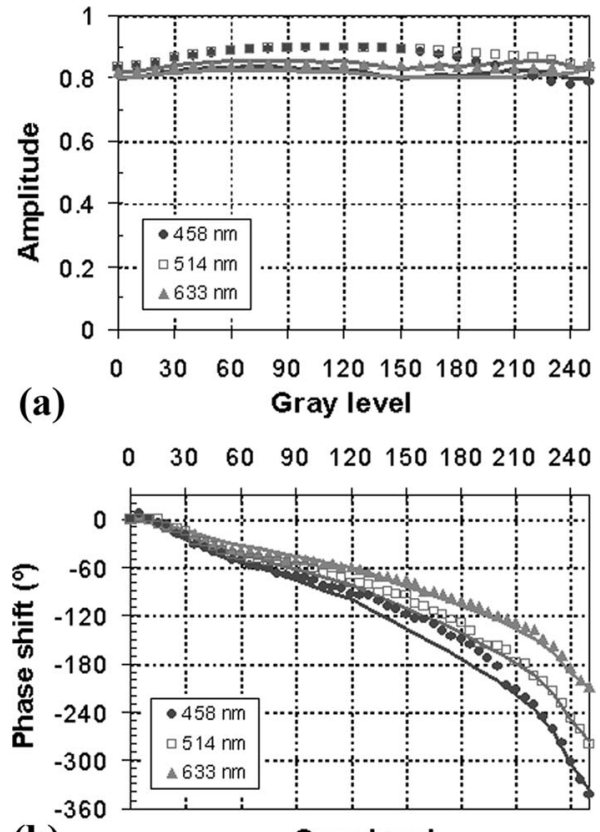

(b)

Gray level

Fig. 1. Complex amplitude modulation for the optimum phase-only regime for the wavelengths 633,514 , and 458 nm: (a) amplitude, (b) phase shift.

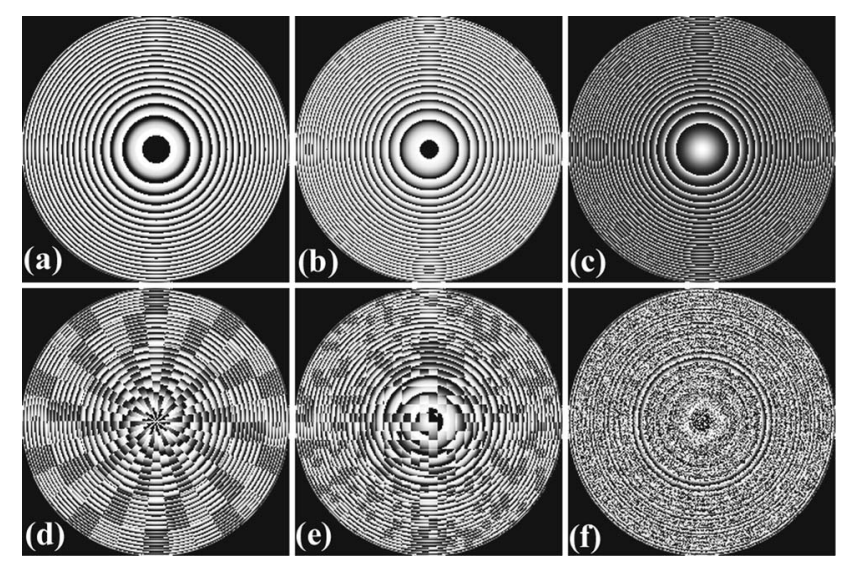

Fig. 2. Gray-level representations of diffractive lenses with equal focal lengths. In (a), (b), and (c) the lenses have been designed for the wavelengths 633,514 , and $458 \mathrm{~nm}$, respectively. In (d), (e), and (f) the lenses are the result of the spatial multiplexing with equal weights of the lenses (a), (b), and (c), with (d) divided into 30 sectors and three rings, (e) random multiplexing in areas of $8 \times 8$ pixels, and (f) random multiplexing in areas of $1 \times 1$ pixel.

The phase-shift dynamic range is close to $360^{\circ}$ at 458 $\mathrm{nm}$, and it is still very high, $210^{\circ}$, at $633 \mathrm{~nm}$.

In Fig. 2 we show gray-level plots of a variety of diffractive lenses designed with the same focal length. Figures 2(a)-2(c) correspond to diffractive lenses designed for monochromatic illumination at 633,514 , and $458 \mathrm{~nm}$, respectively, which hereafter we refer to as the red (R), green $(\mathrm{G})$, and blue (B) channels. One can see that the radii of the circular rings are different in the three cases. Any of these three lenses would show dramatic chromatic focal dependence. We use two spatial multiplexing techniques. In the first, annular-sector multiplexing, the aperture is divided into $N$ rings and $k N$ sectors, where $k$ is a positive integer and $N$ is the number of channels to be multiplexed (three in our case). In the first ring the channels are distributed in an ordered sequence, e.g., RGBRGB ..., where the pixels of the first sector are taken from the lens corresponding to the red channel, the pixels of the second sector are from the green channel, and so on. In the second and the third rings, the channels are shifted (GBRGBR ...), (BRGBRG ...) to make the distribution of the channels more uniform. In Fig. 2(d) an example with three rings and 30 sectors is shown. In the second multiplexing technique, random multiplexing, the aperture is divided into subapertures that are randomly distributed among the three channels. We can change the weight of each channel by varying the probabilities $\left(p_{r}, p_{g}, p_{b}\right)$ with $p_{r}+p_{g}+p_{b}$ $=1$. In Figs. 2(e) and 2(f) examples of the random multiplexing are shown in which the subapertures have sizes of $8 \times 8$ and $1 \times 1$ pixels, respectively. In these figures the three probabilities are the same $\left(p_{r}=p_{g}=p_{b}\right)$. In the experiments several probability combinations have been considered, as we show in Fig. 3.

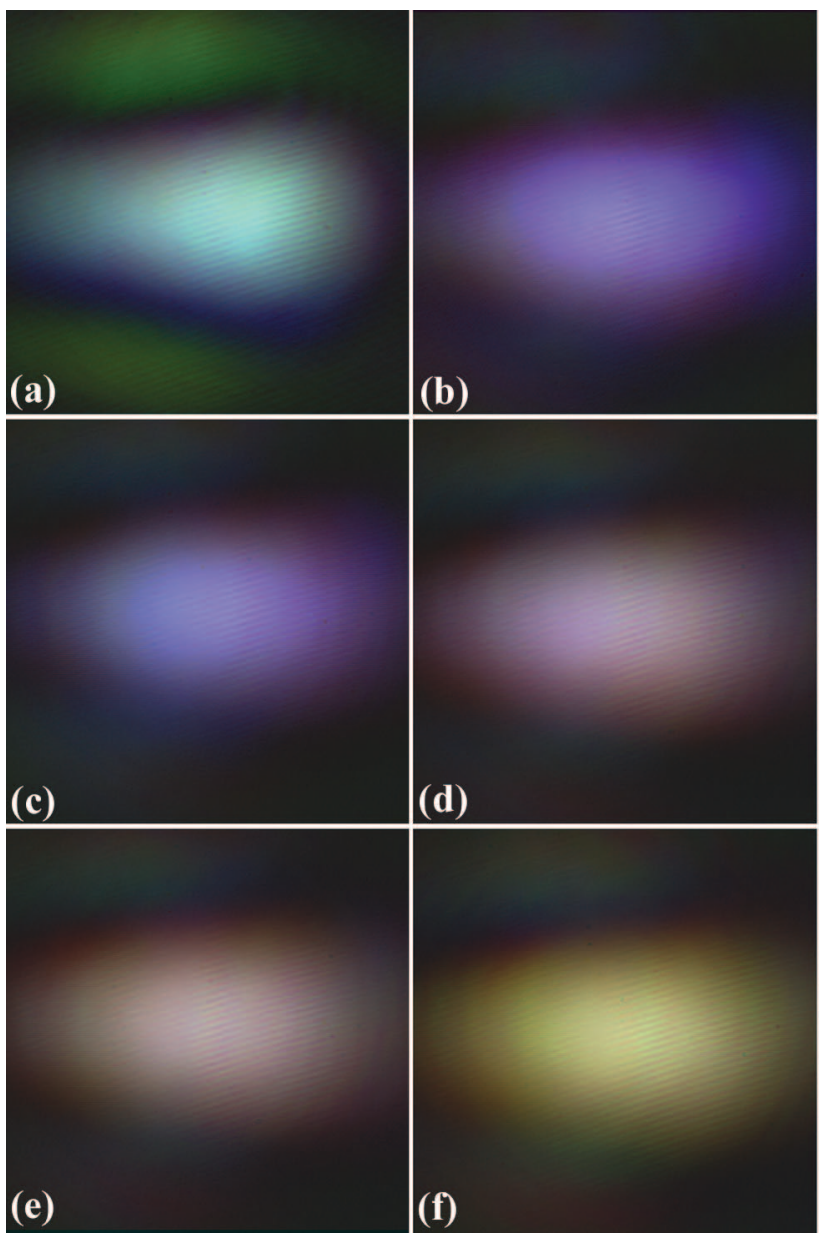

Fig. 3. Pseudocolored PSF obtained experimentally. In (a) and (b) two lenses with weights $(1,1,2)$ for the RGB channels and with different spatial multiplexing schemes are used. (a) 30 sectors divided into three rings, (b) random distribution in $8 \times 8$ pixel areas. In (c)-(f) random multiplexed lenses with $1 \times 1$ pixel areas are used with RGB weights (c) $(1,1,2),(d)(1,1,1.5),(e)(1,1,1.3),(f)(1,1,1)$. 
We note that in principle the multiplexed lenses must produce various monochromatic focalizations: each of the three subapertures will focus each of the three wavelengths used in this work in a different location, thus producing nine monochromatic focalizations. Due to the equal focal length programmed in the three multiplexed lenses, three of these monochromatic focalizations are much more efficient and coincide in the same plane, which is the focus plane of interest for us. The remaining six monochromatic focalizations have a lower efficiency and are defocused, therefore in the focus plane of interest they contribute as a noise background, which as we show in Fig. 3 does not degrade the experimental results obtained.

To evaluate experimentally the performance of the multiplexed lenses we adjust the intensity that is incident on the LCD so that it is equal for the three wavelengths. The point-spread function (PSF) is captured with a monochromatic CCD coupled to a microscope objective to magnify the image. In this work the PSFs for the three wavelengths 633, 514, and 458 $\mathrm{nm}$ are then pseudocolored as RGB channels and combined in a single image. The lenses have a focal length of $1 \mathrm{~m}$ and a diameter of 480 pixels (19.68 $\mathrm{mm}$ ). The incident illumination is collimated so that the programmable diffractive lens is the only one responsible for focusing.

In Figs. 3(a) and 3(b) we show the pseudocolored PSFs produced by two multiplexed lenses both with the same weight distribution $(1,1,2)$ for the RGB channels. Figure 3(a) corresponds to an annularsector multiplexed lens with 30 sectors and three rings, and Fig. 3(b) corresponds to a random multiplexed lens divided into $8 \times 8$ pixel areas. One can see that in Fig. 3(a) there is an intense sidelobe, whereas it disappears with the random multiplexing in Fig. $3(\mathrm{~b})$. We think that the sidelobe is due to the annular structured distribution of the subapertures for each of the three multiplexed lenses, producing a slight hyperresolving effect. One can see that the sidelobe has a different chromaticity compared with the central maximum. In principle, this variation of the chromaticity along the PSF is a nondesired artifact that can be reduced if we decrease the sidelobe. In Fig. 3(b) the random distribution of the pixels makes the subaperture of each of the three multiplexed lenses more uniform, thus avoiding the appearance of high sidelobes. The asymmetry in the PSFs is due to an astigmatic phenomenon in the LCD as was reported in Ref. 6.

In Figs. 3(c)-3(f) we show the PSFs obtained with a random multiplexed lens divided into $1 \times 1$ pixel areas. The RGB weights assigned vary for the four PSFs: $(1,1,2),(1,1,1.5),(1,1,1.3)$, and $(1,1,1)$, respectively, for Figs. 3(c)-3(f). One can see that, as in Fig. $3(\mathrm{~b})$, there is no apparent sidelobe in any of these fig- ures and the chromaticity does not vary markedly along the PSF. With these figures we want to show that by changing the weights for the various channels we can finely tune the chromaticity of the PSF. Toward this goal we have measured the value of the maximum gray level of each of the three RGB components comprising the pseudocolored PSFs. For Figs. $3(\mathrm{c})-3(\mathrm{f})$, these maximum gray-level values are (141, $140,223),(178,164,189),(190,179,173)$, and (209, $216,148)$, respectively, where the three ordered elements in the arrays correspond to the value for each of the RGB component images. One can see that as we go from Figs. 3(c)-3(f) the maximum gray-level value for the B component decreases from 223 to 148. Simultaneously, the maximum gray-level values for the $\mathrm{R}$ and $\mathrm{G}$ components increase. This is consistent with the fact that the weight for the blue subaperture in the multiplexed lens is also decreased, whereas the area occupied by the R and G subapertures increases. We can also say that in each of the four PSFs the values of the maximum gray level for the $R$ and $G$ components are quite similar, which is consistent with the equal weights assigned to the $R$ and $G$ channels in the multiplexed lenses. We want to mention that in the experiments with random multiplexed lenses control of the chromaticity was finer when 1 $\times 1$ pixel areas were used instead of $8 \times 8$ pixel areas.

In conclusion, in this work we have demonstrated a programmable diffractive lens written on a LCD with equal focal lengths for several wavelengths simultaneously. Toward this goal, we achieved a phase-only regime simultaneously for the various wavelengths through an optimization procedure. Among the various multiplexing schemes, random multiplexing provides no sidelobes, and among the random multiplexed schemes we found that the $1 \times 1$ pixel area provided the best tunability for the chromaticity.

We acknowledge financial support from the Spanish Ministerio de Ciencia y Tecnología (grant BFM2003-06273-C02-01/FISI) and from the Generalitat de Catalunya (grant ACI2003-42). C. Iemmi gratefully acknowledges the support of the Universidad de Buenos Aires and CONICET (Argentina). A Márquez's e-mail address is amarquez@dfists.ua.es.

\section{References}

1. V. Laude, Opt. Commun. 153, 134 (1998).

2. E. Tajahuerce, J. Lancis, V. Climent, and P. Andrés, Opt. Commun. 151, 86 (1998).

3. E. Carcolé, M. S. Millán, and J. Campos, Opt. Lett. 20, 2360 (1995).

4. E. Ben-Eliezer, Z. Zalevsky, E. Marom, and N. Konforti, J. Opt. A Pure Appl. Opt. 5, S164 (2003).

5. A. Márquez, C. Iemmi, I. Moreno, J. A. Davis, J. Campos, and M. J. Yzuel, Opt. Eng. 40, 2558 (2001).

6. A. Márquez, C. Iemmi, I. Moreno, J. Campos, and M. J. Yzuel, Opt. Express 13, 2111 (2005). 